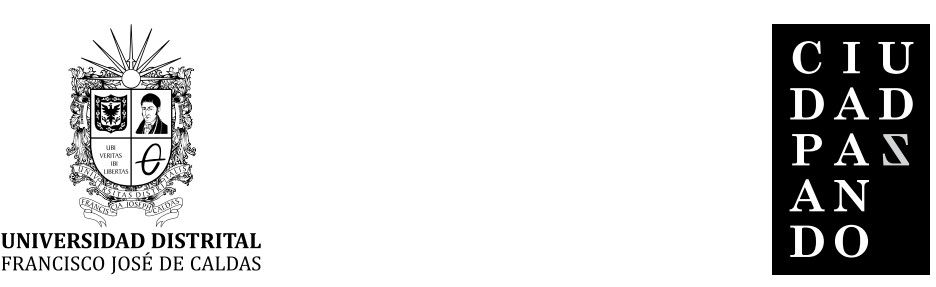

DOSSIER

Artículo de reflexión

\title{
Espacios públicos y posconflicto: lecciones para Colombia
}

Public spaces in post-conflict contexts: lessons for Colombia

Espaços públicos e pós-conflito: lições para Colômbia

\section{Lorena Andrea Erazo Patiño ${ }^{1}$}

\section{Sylvie Nail ${ }^{2}$}

Para citar este artículo: Erazo, L. y Nail, S. (2017). Espacios públicos y posconflicto: lecciones para Colombia. Revista Ciudad Paz-ando, 10.1, 47-58

doi: https://doi.org/10.14483/2422278X.11778

Fecha de recepción: 22 de marzo de 2017

Fecha de aceptación: 14 de julio de 2017

1 Magister en Gerencia para el Desarrollo de la Universidad Externado de Colombia; profesional en Relaciones internacionales y Estudios Políticos, Universidad Militar Nueva Granada (UMNG); investigadora de la Facultad de Educación y Humanidades de la UMNG. Correo electrónico: lorena.erazopatino@gmail.com

2 Doctora en Estudios británicos; profesora de cátedra de la Universidad de Nantes (Francia), Facultad de Idiomas y culturas extranjeras; profesora invitada de la Universidad Externado de Colombia, Facultad de Fianzas, Gobierno y Relaciones Internacionales. Correo electrónico: sylvie.nail@uexternado.edu.co 


\section{RESUMEN:}

Los espacios públicos, especialmente los espacios verdes en los centros urbanos, son lugares privilegiados para favorecer la cohesión social en cualquiera sociedad. En este artículo, por medio de una revisión bibliográfica y del análisis de varios casos de sociedades en posconflicto, se busca entender la importancia de dichos espacios en tal situación. De esta manera, se realiza una aproximación novedosa, puesto que hasta el momento no se había contemplado el papel de los espacios verdes en el posconflicto como herramienta de cohesión social, con el objetivo de proponer estrategias aplicables a este nuevo contexto sociopolítico al que se enfrentará Colombia con la implementación de los acuerdos de paz. Palabras clave: cohesión social, Colombia, espacios verdes urbanos, posconflicto.

Public spaces, especially public green spaces in cities, are privileged areas to promote social cohesion in any society. Through a literature review and through the analysis of various cases of societies in situations of postconflict, this article aims at understanding the importance of such spaces in postconflict contexts. Thus, through a novel approach, because the role of green spaces in post-conflict as a tool of social cohesion had not been contemplated until now, the purpose is to suggest strategies applicable to the new sociopolitical context which Colombia will face with the implementation of the peace agreements.

Keywords: Colombia, post-conflict, public urban green spaces, social cohesion.
Espaços especialmente públicos e espaços verdes nos centros urbanos são locais privilegiados para a promoção da coesão social em qualquer sociedade. Neste artigo, através de uma revisão da literatura e análise de vários casos de sociedades pós-conflito. Assim uma nova abordagem é realiza, já que até agora não tinha sido contemplado o papel dos espaços verdes em pós-conflito como um instrumento de coesão social, afim de propor aplicáveis á este novo contexto sociopolítico para que á Colômbia é confrontado com a implementações dos acordos da paz

Palavras-chave: coesão social, Colômbia, espaços verdes urbanos públicos, pós-conflito. 


\section{Introducción}

El conflicto armado que se ha desarrollado en Colombia durante los últimos cincuenta años se ha caracterizado por ser un fenómeno pluridimensional que se ha transformado de acuerdo con las dinámicas y necesidades de sus actores de manera estratégica, permeando todos los aspectos de la sociedad colombiana: lo económico, lo político, social y hasta lo ambiental (Mantilla, 2012; Lavaux, 2004; Echandía, 2006). Dicha coyuntura no se ha quedado solamente en la esfera rural, sino que también ha impactado profundamente las dinámicas de las ciudades colombianas; las oleadas migratorias del campo a la ciudad resultaron en una gestión del territorio sin planificación y en la creación en la periferia de cinturones de pobreza y segregación que favorecieron la exclusión social de sus habitantes y el deterioro de la seguridad por el incremento de delitos comunes (Ruiz, 2009).

La problemática del presente trabajo se enmarca en un contexto doble: en primer lugar, el acuerdo general para la terminación del conflicto y la construcción de una paz estable y duradera — que se firmó en Bogotá el 26 de noviembre de 2016-; y en segundo lugar, en un contexto más general que se puede forjar en las ciudades luego de la terminación de un conflicto armado. Ordóñez y Duinker (2014) resaltan la complejidad de tal situación, afirmando que para la comunidad internacional y el mismo Estado en transición implica un escenario difícil para todos los actores, no solamente por el hecho de tratar de evitar que la violencia se reviva, sino también de que se restablezcan las relaciones internas en un camino pacífico.

Lo anterior se explica por la fractura del tejido social y la integración a las ciudades de comunidades desplazadas por la violencia o la reintegración a la vida civil de excombatientes. Si no se atienden estas situaciones de manera adecuada por parte del gobierno, estas situaciones pueden generar un caos en materia de seguridad, afectando sustancialmente la calidad de vida de los ciudadanos, como se evidenció en otros países. De dichas experiencias surge la necesidad de nuestra reflexión en relación con la situación actual en Colombia, donde se puede esperar que se dé una transformación de la violencia política de más de 50 años en una violencia común con una presencia significativa en los centros urbanos.

Otro reto al que se enfrenta la sociedad colombiana y que los investigadores han ignorado típicamente, es la influencia de las cuestiones ambientales en los acontecimientos sociales y políticos en los países en posconflicto. Como afirma Krampe (2016) "los vínculos de los procesos sociales, políticos y ambientales siguen siendo rudimentarios". Es en esta perspectiva particular y novedosa que este artículo se enmarca.

El desarrollo del presente trabajo es el resultado de estudios sobre dos temas. Primero, la experiencia de otros países en situaciones de posconflicto deja pensar que, si no se contempla en el plan de implementación de los acuerdos de paz unas herramientas concretas para facilitar el proceso de reconciliación nacional y reconstrucción de tejido social, se puede generar un fracaso o por lo menos unos retrasos importantes en materia de seguridad, cohesión social e institucionalidad. Segundo, la paz supone una cohesión social, que necesita vínculos estrechos con el territorio y, siendo los centros urbanos los lugares mayoritariamente habitados y en donde se encuentran diferentes comunidades y culturas, se requieren espacios públicos que reflejen la diversidad, la equidad e igualdad entre los ciudadanos, que permitan la identificación con su cultura y propugnen un sentido de pertenencia.

Para tal fin, primero se revisan las investigaciones que demuestran la utilidad de los espacios públicos de naturaleza en las ciudades para generar identidad y cohesión social. En segundo lugar, se analizan las experiencias internacionales en sociedades en posconflicto en torno a los espacios públicos. Finalmente se proponen estrategias que podrían servir como lineamientos de políticas públicas relacionadas con el posconflicto en Colombia.

De esta manera, el análisis realizado en el presente artículo se enmarca en los estudios de desarrollo, pues dada su característica multidisciplinar permite abordar la problemática de manera integral, orientando el estudio hacia la búsqueda de un estado de bienestar dentro de las dinámicas estructurales y a su vez complejas de una sociedad en transformación.

\section{Marco conceptual}

\section{Relación entre posconflicto y cohesión social}

El termino posconflicto es usado comúnmente en las ciencias políticas, económicas y en la literatura sobre desarrollo para referirse a una sociedad que recientemente ha experimentado un conflicto violento que afectó la vida diaria de la mayoría de sus ciudadanos (Chetail, 2009). Al posconflicto también se asocian otros elementos conceptuales que permiten una transición estable hacia la paz: una de ellas es la consolidación de la paz o peacebuilding, considerado como una serie de medidas para identificar y apoyar estructuras que tiendan a fortalecer y solidificar la paz a fin de evitar una recaía en el conflicto (ONU, 1992).

Las medidas que se deben tomar en las sociedades en posconflicto para la construcción de paz abarcan, primero, las orientadas a limitar el impacto de la violencia armada y de sus consecuencias más directas; segundo, las actividades encaminadas a construir una paz duradera, es decir, las que se encuentran directamente relacionadas con las causas del conflicto y por lo tanto tienen un desarrollo a largo plazo; y tercero, las que facilitan los medios para hacer posible una transformación no violenta del conflicto (l'Escola de Cultura de Pau, s.f).

En este sentido, las actividades relacionadas deben forjar de manera progresiva la consolidación de todas las estructuras creadas para facilitar la transición y el 
desmonte de las barreras que eviten los posibles retrocesos. Si bien los indicadores de éxito de la construcción de paz aún no están claramente establecidos por los diferentes centros de estudios, sí hay una clara tendencia, en que las medidas tomadas deben tener un reflejo en la reducción de homicidios (Rettberg, 2013).

Experiencias internacionales de posconflicto han dejado en evidencia que los procesos de construcción de paz no son sencillos ni son regulares en su evolución (Rettberg, 2013). Se deben plantear estrategias realistas para que la reducción de la violencia política no aumente la violencia común de manera exponencial, ni que la sensación de inseguridad en la sociedad mine la calidad de vida de los ciudadanos (Dawson, 2006). Indudablemente, el conflicto colombiano tiene características particulares que hace difícil una comparación con otros conflictos y no se pretende analizar el desarrollo de los conflictos ni sus particularidades, sino que se parte del hecho de que son sociedades en situaciones de posconflicto. No obstante, con el ánimo de abrir nuevos caminos de análisis a partir de las experiencias existentes se seleccionaron casos como El Salvador, Sudáfrica e Irlanda del Norte, a fin de realizar un breve estudio del desarrollo del posconflicto, para el propósito que se presentará más adelante.

De otro lado, la cohesión social, aunque aplica a todas las sociedades, es otro elemento conceptual relacionado con situaciones de posconflicto. En el contexto latinoamericano específicamente, por ser la región con más desigualdad, la cohesión social es entendida como "la capacidad de las instituciones para reducir de modo sustentable las brechas sociales con apoyo ciudadano" (Buonomo y Yanes, 2013).

Otros investigadores han redefinido el concepto de cohesión social en términos de las interacciones tanto horizontales como verticales entre los miembros de una sociedad, que implican confianza, sentido de pertenencia y la voluntad de participar y ayudar, así como sus manifestaciones conductuales (Chan, To and Chan, 2006). La anterior definición es la que se ajusta en mayor medida al tipo de análisis que se realiza en el presente artículo, sobre todo en lo que atañe a los espacios verdes públicos.

Se ha utilizado el término cohesión social como variable determinante en estudios sobre crimen y violencia, la hipótesis que más tiene respaldo por investigadores como Forrest y Kearns (2001) es la correlación entre la falta de cohesión social y los altos niveles de crimen y violencia en las comunidades. Dicha correlación es también aplicable a las sociedades en postconflicto, puesto que la fragilidad social luego de los conflictos armados es más alta y se requiere un trabajo articulado por parte de la comunidad y del Estado por medio de programas educativos en ciudanía que involucren la cohesión social.

\section{Espacios públicos}

El espacio público como dimensión espacial de la ciudad y lugar de identificación social de sus habitantes, ha sido tema de interés de varios filósofos y autores a través del curso de la humanidad, desde Aristóteles hasta Hannah Arendt. Así, el alma que da vida a las ciudades es el conglomerado de personas que la habitan y las actividades que se desarrollan a diario en sus calles, parques, plazas, etc. y que contribuyen significativamente al crecimiento tanto físico como social de la ciudad. Por ejemplo en Grecia, el ágora ateniense fue el hito inaugural del espacio público como ideal democrático, donde se realizó la primera distinción entre lo público y lo privado dentro del marco de la configuración de las ciudades-Estado (Berroeta y Moranta, 2012). Ferry (1995) afirma que el concepto de ágora es tomado como el lugar donde los ciudadanos deben reunirse a debatir sobre asuntos concernientes al gobierno de la ciudad y donde son libres de las obligaciones cotidianas. Es decir, que lo público se equiparó a lo políti$\mathrm{co}$, donde las decisiones tomadas en este espacio estaban dotadas de legitimidad para regular la convivencia entre los ciudadanos.

En su obra la Politica (1932), Aristóteles medita sobre el beneficio de la unidad en la ciudad, llegando a la conclusión de que al hacerse más unitaria ya no sería ciudad, puesto que el concepto de ciudad está estrechamente relacionado con la pluralidad, sino que debe existir un equilibrio entre unidad y pluralidad. Para Habermas,

la ciudad es sobre todo el espacio público donde el poder se hace visible, donde la sociedad se fotografía, donde el simbolismo colectivo se materializa. La ciudad es un escenario, un espacio público que cuanto más abierto esté a todos, más expresará la democratización política y social. (en Borja y Muxi, 2003)

Por su parte, Hannah Arendt lo entiende como:

un ámbito que se conforma cuando las personas, concibiéndose como igual, se reúnen para actuar y dialogar en concierto [...] caracterizado por: (i) que las personas se reúnan concibiéndose como iguales (isonomía e isegoría), (ii) para dirimir sus asuntos fundamentalmente a través de la acción y de las palabras, y (iii) que esto sea visible y accesible -formalmente- para todos. (Di Pego, 2006)

Las definiciones expuestas destacan la relación estrecha entre: sociedad —espacio público- ciudad, en la cual el espacio público se convierte en la expresión misma de la sociedad. Por lo tanto, el espacio público puede estar definido no solo por su libre acceso y uso, sino por el conceso generado en torno al tipo de manifestaciones sociales que se pueden desarrollar en estos espacios (Neira, 2007). En torno a este enfoque del espacio público se desarrolla el análisis propuesto, el cual 
tiene una perspectiva social y una visión funcional como articulador de los procesos sociopolíticos; es allí, donde se otorga legitimidad a las decisiones tomadas en la esfera política y se materializan en los espacios comunes para todos los ciudadanos, siendo una expresión misma de la democracia.

\section{Espacios públicos verdes urbanos}

Los espacios verdes constituyen un tipo particular de espacio público. De manera más precisa, se caracterizan por ser espacios públicos compuestos principalmente por vegetación, que se encuentran en óptimas condiciones para la recreación y esparcimiento y brindan seguridad a los usuarios (Guerrero y Culós, 2007). Carmona (2010) los define como zonas abiertas y accesibles con árboles individuales, espacios diseñados de menor tamaño y escenarios naturales conectados con las zonas construidas.

Los espacios verdes públicos ofrecen servicios individuales relacionados con la salud, satisfacción espiritual, servicios recreacionales, de carácter estético y con la sensación de confort y calidad de vida en las ciudades (Millennium Ecosystem Assessment, 2003). Además de los beneficios individuales, una literatura voluminosa multidisciplinaria ha demostrado en los últimos 30 años los aportes de los espacios verdes urbanos a la cohesión social.

A partir de una extensa revisión de literatura y de estudios de caso, Kazmierczak (2010) explica de cuáles maneras los espacios verdes aportan a la cohesión social, lo que corrobora las teorías expuestas anteriormente sobre los espacios públicos en general. Primero, son equipamientos de acceso libre y gratuito cerca de donde viven los citadinos. Segundo, por esta misma razón, son escenarios que permiten y facilitan las interacciones sociales. Pero, además de eso, por ser lugares que facilitan el contacto con la naturaleza, alivian el estrés y la agresividad. Y, finalmente, si están en un buen estado de mantenimiento, estos espacios verdes tienen el potencial de facilitar la identificación de la ciudadanía con un territorio.

Por lo tanto, se justifica plenamente el acento puesto, dentro de la Nueva Agenda Urbana adoptada durante la conferencia Hábitat III en octubre de 2016, sobre el espacio público como un espacio multifuncional que permite el desarrollo y bienestar de las sociedades. En el artículo 37, los Estados firmantes se comprometen a

promover, espacios públicos inclusivos, accesibles, verdes y seguros y de calidad, incluyendo calles, aceras y carriles para bicicletas, plazas, áreas frente al mar, jardines y parques, que son áreas multifuncionales para la interacción social y la inclusión, la salud humana y el bienestar siendo, el intercambio económico, y la expresión cultural y el diálogo entre una amplia diversidad de personas y culturas, y que están diseñados y manejados para asegurar el desarrollo humano y la construcción de sociedades pacíficas, incluyentes y participativos, así como para pro- mover una vida juntos, conectividad e inclusión social.

(United Nations, 2016) [énfasis agregado]

Este compromiso resalta la importancia de los espacios verdes públicos como una herramienta inclusiva y multifuncional, que se relaciona con la percepción de igualdad en los espacios públicos que plantea Hannah Arendt.

En Colombia, la definición actual y oficial del espacio público se encuentra en el artículo 139 del reciente Código Nacional de Policía y Convivencia (2017) e incluye, entre otros, "humedales", "rondas de los cuerpos de agua, parques, plazas, zonas verdes y similares", "los elementos históricos, culturales, religiosos, recreativos, paisajísticos y artísticos”, “y, en general, todas las zonas existentes y debidamente afectadas por el interés colectivo manifiesto y conveniente y que constituyen, por consiguiente, zonas para el uso o el disfrute colectivo".

El término "espacios públicos" se usará en este estudio únicamente para referir a los espacios públicos verdes urbanos (parques de pequeño, mediano y grande tamaño, bosques urbanos), que son los que más han sido estudiados en este contexto a nivel internacional, con la intención de establecer los lineamientos de la política pública en el tema para Colombia.

\section{Metodología}

Una revisión bibliográfica demuestra la existencia de numerosos trabajos investigativos que concluyen en el papel sobresaliente de los espacios públicos para contribuir a un tejido social sólido (Kazmierczak, 2010; Peters, Elands, and Buijs, 2010). Entre ellos uno elaborado en Colombia por Ordóñez y Duinker (2014) sobre el urbanismo social en Medellín integra las variables de análisis de espacios públicos y cohesión social, mas no contempla la dimensión del posconflicto. Las investigaciones resaltan desde hace más de tres décadas la importancia de los espacios de naturaleza urbanos para generar beneficios múltiples relevantes a la cohesión social (Borja and Muxi, 2003; Segovia, 2007; Burbano y Páramo, 2014). Pero ninguno está enfocado a estudiar el papel del uso de los espacios verdes existentes para facilitar la cohesión social y la reconciliación nacional en casos de posconflicto.

A partir de los argumentos expuestos en la sección anterior, se formula entonces la hipótesis de que los espacios públicos son lugares donde se pueden desarrollar estrategias eficaces para la construcción de ciudadanía y la consolidación de la paz en un escenario de posconflicto. En consecuencia, se quiere interrelacionar las variables de reducción de la violencia y la criminalidad, espacios verdes y cohesión social, lo cual permitirá dentro de las limitaciones mencionadas extraer lecciones que pueden servir en Colombia.

Por lo tanto, para comprobar la hipótesis planteada se analizan tres casos internacionales de sociedades con 
proceso recientes de posconflicto: El Salvador, Sudáfrica e Irlanda del Norte; la selección de estos casos obedece a la misma diversidad y complejidad de cada proceso, lo cual permite tener una perspectiva ampliada para cada una de las variables en el escenario mencionado. Adicionalmente, se escogieron algunos casos desarrollados en Colombia, en Medellín, Bogotá y el Urabá antioqueño; puesto que, a pesar de estar en medio del conflicto deja en evidencia la importancia de los espacios públicos verdes para la construcción de paz. Finalmente, para la construcción de una estrategia para Colombia se destaca la naturaleza de las iniciativas dentro del análisis de los modelos top-down y botton-up usados en las ciencias sociales, con relación a la forma en que se implementan las estrategias en cada caso.

\section{Resultados}

\section{Importancia de la cohesión social en el posconflicto}

A raíz del prolongado conflicto colombiano, la sociedad en Colombia se resquebrajó de tal manera que hizo que se transformara en una sociedad tolerante a los diferentes tipos de violencia y enferma de anomia ${ }^{3}$ (Reyes Morris, 2016).

Desde esta perspectiva, la paz se convierte en un reto de gran magnitud para el país, puesto que se debe redefinir el contrato social en un escenario de extrema fragilidad y requiere de la articulación de los diversos actores involucrados para lograr un proceso exitoso. Como bien lo dice Rettberg (2013), más allá de la desmovilización de los combatientes, una "paz estable y sostenible requiere de una reducción de los homicidios, pero apalancada por cambios sociales, económicos y políticos cruciales".

La bibliografía relacionada con el posconflicto encuentra como característica común que la violencia no conlleva una reducción real en este nuevo escenario (Steenkamp, 2005, 2011; Schuld, 2013; Boyle, 2015, en Camelo, 2015) y luego de los acuerdos de paz, suelen relacionarse directamente con la criminalidad y dejan al descubierto los conflictos sociales ocultos bajo la violencia política (Camelo, 2015). Por ejemplo, en Sudáfrica, una vez se dio la transición a la democracia, después de la firma de los acuerdos de paz, la desigualdad entre las comunidades negras se incrementó y la cohesión social se debilitó significativamente, afectando los índices de criminalidad y violencia de manera especial en las ciudades (Veit, Barolsky and Pillay, 2011). Según Palmary (2015), las formas en que se ha adoptado la cohesión social en

3 De acuerdo con el Doctor Reyes Morris (2016) la anomia es una situación social en la cual las normas estipuladas ya no tienen la suficiente fuerza para regular las diferentes interacciones de la cotidianidad social, es decir que se ha dado una pérdida de legitimidad. También se entiende como una inestabilidad y desintegración del tejido social.
Sudáfrica evidencian un trato como sinónimo de construcción de nación: tal es el caso del título del plan de desarrollo nacional, A National Strategy for Developing an Inclusive and Cohesive South Africa, en donde se evidencia esta concepción de la construcción de paz al equiparar los términos.

Dicha coyuntura también ocurre en El salvador: a pesar de la implementación de políticas públicas que buscan fortalecer la cohesión social, estimular la participación ciudadana por medio de la promoción de valores, principios y convicciones democráticas con el fin de prevenir la violencia, así como fortalecer las instituciones involucradas para favorecer los procesos administrativos relacionados (OPAMSS, S.f.), no se ha logrado un impacto contundente. Además del fenómeno de violencia de las Maras, se dejó de lado el papel protagónico de la sociedad salvadoreña y no se buscó sanar las heridas sociales, ni restablecer el tejido social.

Los lugares públicos se volvieron símbolos de la desconfianza generalizada. Según una encuesta de 2009, Figura 1, entre los lugares percibidos como de mayor inseguridad, figuran en tercer puesto las plazas públicas y parques (Jiménez, 2010).

\begin{tabular}{|l|l|}
\hline \multicolumn{2}{|c|}{ Lugares percibidos como de mayor inseguridad } \\
\hline Dónde siente más inseguridad & Porcentaje \\
\hline El autobús & $90.0 \%$ \\
\hline El mercado & $80.5 \%$ \\
\hline Las plazas Públicas y parques & $78.0 \%$ \\
\hline El centro de la ciudad donde vive & $67.1 \%$ \\
\hline IDUOP, 2009. & \\
\hline
\end{tabular}

Figura 1. Lugares percibidos como de mayor inseguridad. Fuente: Jiménez (2010, p.42).

En consecuencia, el $63 \%$ evitan ciertos lugares de recreación, Figura 2.

\section{Reacción ciudadanía ante la delincuencia}

\begin{tabular}{|l|l|}
\hline Cambios de conducta y actitud & Porcentaje \\
\hline Evitar visita a lugares de recreación & $63.1 \%$ \\
\hline Reducir lugares donde van de compras & $53.2 \%$ \\
\hline Cierre de negocios a causa de la delincuencia & $22.5 \%$ \\
\hline Necesidad de irse del barrio donde viven & $19.6 \%$ \\
\hline Organizarse entre los vecinos para protegerse & $17.3 \%$ \\
\hline Piensan irse del país por temor a la delincuencia & $16.9 \%$ \\
\hline Cambiar el número telefónico debido a las amenazas & $10.9 \%$ \\
\hline IUDOP, 2009. &
\end{tabular}

Figura 2. Reacción ciudadana ante la delincuencia

Fuente: Jiménez (2010, p. 47). 
Este contexto llevó en 2015 a El Salvador a ser el país más violento del hemisferio occidental, teniendo la tasa de homicidios más alta. Dentro del Plan quinquenal de desarrollo 2014- 2019, se reconoce la limitación institucional en términos de capacidades para responder a las problemáticas de espacio público, seguridad y violencia, debido a la falta de una política integral y a la coordinación entre las diversas instituciones y entidades.

De la misma manera, en Irlanda del Norte, durante el conflicto entre las comunidades protestante y católica, los espacios públicos se volvieron escenarios de violencia, a tal punto que se construyeron muros (denominado peacelines) en distintos barrios para evitar los enfrentamientos. Uno de estos peacelines fue edificado en Alexandra's Park en Belfast y lo cortó en dos. En visa de este contexto, después del acuerdo de paz firmado en 1998, la agenda de inclusión y cohesión social realizada por el Gobierno se basó en tres estrategias claves: los programas de SEUPB PEACE, las políticas de un futuro compartido y cohesión e intercambio e integración (Graham, 2012).

En síntesis, las experiencias muestran que la construcción de la paz genera retos que no siempre se anticipan y exigen a los estados, a la ciudadanía y a la comunidad internacional, trabajar en estrategias alternativas que lleven implícitas un carácter multidimensional y holístico, para fortalecer la cohesión social, restablecer la confianza, dignificar a las víctimas y sobre todo darle más legitimidad al Estado y sus instituciones.

\section{El papel de los espacios verdes urbanos para la cohesión social en casos de posconflictos}

Los espacios públicos se integran perfectamente en tales estrategias: de la misma manera que se prestan para ser escenarios de violencia, los parques y bosques urbanos pueden ser parte de las estrategias de solución en sociedades en posconflicto. Así, en Alexandra's Park en Belfast, después de 1998, una puerta se abrió en el peaceline, que se queda abierta durante el día, como un símbolo de la reconciliación. Aún más, recientemente, un grupo de mujeres han introducido unas bancas en el parque, para que los habitantes se puedan quedar más tiempo e intercambiar.

En El Salvador, a raíz de las observaciones mencionadas y de la falta de convivencia en los lugares de esparcimiento, se ha hecho una importante inversión en infraestructura en 56 comunidades, que incluyen "mejoras en áreas verdes y parques, construcción y equipamiento de complejos deportivos, iluminación de canchas de futbol, juegos infantiles" (Jiménez, 2010). Paralelamente a estas iniciativas top-down, se han desarrollado algunos proyectos de construcción de paz en los espacios públicos, liderados por la sociedad civil, tales como el "monumento a la memoria y la verdad", construido en 2003 en el parque Cuscatlán en San Salvador. Se trata de un muro de 85 metros de largo y 3 metros de alto, donde se hallan consignados los nombres de más de 25626 víctimas, lo que ha permitido que los familiares tengan un espacio de duelo de sus seres queridos y que se genere una apropiación de un espacio subutilizado.

Otra experiencia significativa se desarrolla en el municipio Sonsonate. El proyecto "Tardes de Convivencia" tiene como objetivo desde 2011 "provocar espacios de diversión y sano esparcimiento en la comunidad, en los cuales también se puedan utilizar para llevar mensajes de convivencia pacífica" (Amuprev, S.f). En los espacios públicos en diferentes barrios se realizan actos artísticos y culturales con un mensaje de convivencia pacífica ciudadana. El índice de homicidios en las zonas intervenidas se ha reducido en un 33\% y los robos y hurtos en un $45 \%$. Adicionalmente, este proyecto ha recobrado la confianza de la comunidad en la Policía Municipal, ha creado espacios de acercamiento y reconocimiento entre los actores comunitarios y se lo ha apropiado la ciudadanía, pasando del liderazgo de la alcaldía al liderazgo de la comunidad.

La Republica de Sudáfrica se ha enfrentado a grandes retos en materia de reconciliación y cohesión social después de 1994 (Tepper, S.f), pero existen pocas iniciativas donde se involucra la naturaleza urbana como herramienta de desarrollo social, en su gran mayoría con un enfoque top-down. Entre ellas existe un plan municipal de desarrollo espacial de ciudad del Cabo (Williams, 2000), en donde se busca crear infraestructura verde para los ciudadanos. Por otra parte, existe un programa comunitario del trabajo (Masuku, 2015), donde los ciudadanos en paro tienen un subsidio por 100 días que debe ser condonado con trabajo social en diferentes actividades, una de ellas siendo el cuidado de los parques y espacios verdes. Finalmente, existen otras iniciativas enmarcadas en el modelo bottom-up, en diferentes ciudades de Sudáfrica (Kgalema, 1999) bajo la forma de la construcción por la misma sociedad civil de monumentos en espacios públicos, que los han convertido en zonas conmemorativas para honrar a las víctimas de la violencia política y servir como símbolo del proceso de reconciliación.

Sin embargo, aún no se han desarrollado políticas específicas para promover e integrar el uso de los espacios públicos verdes y, de esta manera, aprovechar sus múltiples beneficios que se verán traducidos en la calidad de vida de sus ciudadanos y la cohesión social urbana, como se encuentra definida en el marco conceptual. Por otro lado, deja en evidencia la complejidad de la aplicación de las políticas públicas en las sociedades en posconflicto, donde se observa una coexistencia, a menudo desarticulada, de los modelos bottom-up y top-down.

En Colombia, cabe resaltar la experiencia del barrio Santo Domingo Savio, ubicado en la Comuna uno de Medellín. Nació como producto de la violencia de los años 60 y 70 por invasión de las poblaciones desplazadas que huían a Medellín en búsqueda de seguridad y oportunidades de vida; este barrio se ha caracterizado por ser un referente de marginalidad, deuda social y altos índices de violencia 
delincuencial, sicariato y narcotráfico, entre otros males sociales. En las últimas décadas, las diferentes administraciones locales, aplicando el concepto de urbanismo social, ${ }^{4}$ sumado al respaldo de la comunidad, han intervenido este sector con infraestructura pública, dotándolo con la biblioteca España, metro cable, parque público, calles etc., como estrategia para contrarrestar la violencia urbana justificada por las condiciones de inequidad en la construcción de ciudad (González y Carrizosa, 2011).

A partir de los casos mencionados y no obstante las pocas situaciones existentes donde se relacionen los espacios verdes de manera significativa, dichas experiencias dejan pensar que, a pesar de la poca presencia estatal en las zonas vulnerables y la falta de herramientas que tienen las instituciones gubernamentales en los casos donde la hay, existe un gran potencial de los espacios verdes para ser aprovechados en las ciudades colombianas y sus nuevas realidades.

\section{Forjando cohesión social en los espacios públicos en medio del conflicto}

En Colombia, antes de las negociaciones de paz, ya se desarrollaban iniciativas ciudadanas y proyectos institucionales que tenían como eje central la cohesión social y, a pesar de que no se desarrollaran de manera consciente en torno a los espacios verdes, sí se consideran estos espacios como herramientas para facilitar el encuentro ciudadano y construir la paz. Se resaltan algunos de esos en este texto, que pueden empezar una lista de buenas prácticas para Colombia en el contexto actual.

El parque de la Bailarina, antiguamente conocido como el parque Astorga en Medellín, surgió tras el feminicidio de Isabel Restrepo Cárdenas, bailarina profesional, en el 2008. Su madre y unos amigos de la artista, de manera espontánea, se empezaron a reunir en el lugar de su deceso y a realizar actividades culturales. Así, nace la corporación Alas de Mariposa en 2011, quienes convocan a las personas a realizar intervenciones artísticas en el espacio público con un mensaje doble: rechazar la violencia y crear conciencia acerca de la necesidad de apropiarse de los espacios públicos como una medida de seguridad, y así concebir un sentido de pertenencia y de cultura ciudadana, que fortalezca la construcción de paz en los entornos urbanos (Corporación Alas de Mariposa, S.f). Esta experiencia refleja la potencialidad de éxito de los procesos bottom-up ya que están dotados de mayor legitimidad al responder a las necesidades específicas de las comunidades afectadas.

4 "El urbanismo social busca transformaciones profundas en las relaciones administración local-ciudadanía a través del uso de la arquitectura, la educación, la recuperación de espacios públicos, el fortalecimiento de la cultura ciudadana, la participación y la confianza en las instituciones gubernamentales para transformar las relaciones que, históricamente, han estado marcadas por las desigualdades sociales y la ausencia estatal”. (González y Carrizosa, 2011)
Un segundo caso se evidencia en Bogotá D.C. con una iniciativa top-down por parte de la Alcaldía mayor, con el programa "Paz a la paz". Este programa surgió dentro del marco de la política Bogotá Humana y contó con un trabajo artículo con otras organizaciones deportivas y organizaciones de cooperación internacional (PNUD). Busca promover los valores deportivos como la fuerza, el trabajo en equipo y la solidaridad para fomentar hábitos de convivencia, reconciliación y paz en poblaciones vulnerables de los colegios distritales de la ciudad. Dentro de los compromisos establecidos en el acuerdo firmado por las instituciones vinculadas se estable específicamente la voluntad de "recuperar espacios, escenarios deportivos y parques para la práctica recreativa para erradicar prácticas delictivas” (IDRD, 2015). Tiene un impacto significativo en la población, puesto que llega a beneficiar a 70000 estudiantes de colegios distritales, busca desarrollar actividades deportivas y recreativas en los 5029 parques de Bogotá de distinta clasificación y dimensión a través de más de 1000 eventos que pretenden sensibilizar a la comunidad, promover la sana convivencia y difundir una pedagogía de paz (IDRD, 2015).

El tercer ejemplo se desarrolla en el Urabá antioqueño, una región reconocida por su contexto de violencia permanente, con los $\mathrm{PMI}^{5}$ que dotan de infraestructura urbana ecológica a la ciudad de Turbo bajo un modelo top-down. En esta zona geoestratégica de importancia por sus "altos índices de biodiversidad, su abundante oferta hídrica, su localización y su extensa zona costera sobre el mar Caribe" (Orsini y Montoya, 2016), se recuperó el caño Veranillo en Turbo y se realizó una gestión de la biodiversidad orientada a la articulación de la vegetación con la oferta de espacios públicos verdes, al favorecer la apropiación de los espacios públicos por medio del deporte y la cultura, propiciando la cohesión social.

\section{Discusión: pistas para Colombia}

A partir de lo anterior se puede contemplar el papel de los espacios verdes urbanos para contribuir a la reconciliación en el caso colombiano. En su estudio sobre el desplazamiento y las identidades campesinas, Osorio afirma que

la llegada a un nuevo territorio requiere un proceso de reestructuración de redes de acción colectiva en nuevos campos de poder, y de resistencia y de solidaridad que se redefinen a partir de la acción y el encuentro con otros. (2007)

5 Planes Municipales Integrales (PMI) son una herramienta de planificación territorial los cuales fueron construidos a partir de una visión holística del territorio donde se involucran activamente factores como la sociedad y la biodiversidad, con la intensión de que los centros urbanos de los municipios del Urabá (Chigorodó, Carepa, Turbo y Apartadó) tengan un crecimiento controlado y donde se integran los espacios verdes a las dinámicas de los ciudadanos. 
Así, la identificación con los espacios surge por medio de la práctica diaria de habitar un lugar donde se configuran prácticas concretas y simbólicas que permiten forjar identidades colectivas y renovar el patrimonio social, a partir de la confianza, la memoria y las historias comunes.

Retomando el caso del barrio Santo Domingo Savio de Medellín y siguiendo lo planteado por Ballesteros, Velásquez, Sierra, Vásquez, y Vélez (2011), es el Estado quien, a través de la intervención socio espacial, recobra la legitimidad perdida por la violencia y, "a su vez, se implementa el espacio público como ámbito generador de interacciones entre la ciudadanía y los múltiples actores, como nuevos espacios de encuentro ajenos a la clandestinidad".

Para que los espacios verdes urbanos jueguen su papel en pro de la cohesión y la reconciliación en Colombia, es imprescindible que se cuiden estos espacios (mantenimiento y seguridad). En Colombia, en los acuerdos de paz con las FARC, las ciudades y los temas urbanos como entornos de implementación de los mismos no se tienen en cuenta. Las instituciones que tienen una relación directa con los temas ambientales a nivel local y nacional (Instituto Humboldt, Ministerio de Ambiente y Desarrollo Sostenible, Departamento Administrativo de Medio Ambiente, Corporaciones Autónomas Regionales etc.), aún no han considerado de manera directa las ciudades como escenarios de posconflicto. Actualmente, se están desarrollando las temáticas ambientales en el marco de los acuerdos de paz a escala rural. En cuanto a las ciudades, hasta ahora se están entablando las conversaciones interinstitucionales para construir la agenda (Montoya, 2017).

A lo anterior se suma el déficit de espacio público en Colombia. Según el Conpes 3718 sobre espacio público, ${ }^{6}$ se establece que, para el año 2010, la superficie por habitante fue de $3,3 \mathrm{~m}^{2}$ lo que representa un déficit de espacios públicos respecto al índice de $15 \mathrm{~m}^{2}$ por habitante de Espacio Público Efectivo ${ }^{7}$ establecido en el decreto 1504 de 1998 (CONPES, 2012), Figura 3.

Uno de los objetivos del presente artículo era abrir un debate en torno a los espacios verdes que permitiera establecer unas recomendaciones para Colombia en el tema, y así facilitar la transición a una sociedad en posconflicto. Por lo tanto, se propone lo siguiente: se debería poner en la agenda pública la relevancia de las ciudades para la construcción de la paz; pues si bien la mayoría de la población colombiana se encuentra concentrada en el $0,36 \%$ del territorio colombiano (IGAC, 2014), esto se

$6 \quad$ Ley $9^{\text {a }}$ de 1989 y el Decreto 1504 de 1998 definen el espacio público como el "conjunto de inmuebles públicos y los elementos arquitectónicos y naturales de los inmuebles privados, destinados por su naturaleza, por su uso o afectación a la satisfacción de necesidades urbanas colectivas que trascienden, por tanto, los límites de los intereses individuales de los habitantes" (Artículos $5^{\circ}$ y $2^{\circ}$, respectivamente) (CONPES, 2012).

7 Se contabilizan varios tipos de espacios públicos tanto verdes como duros dentro del indice de Espacio Público Efectivo.

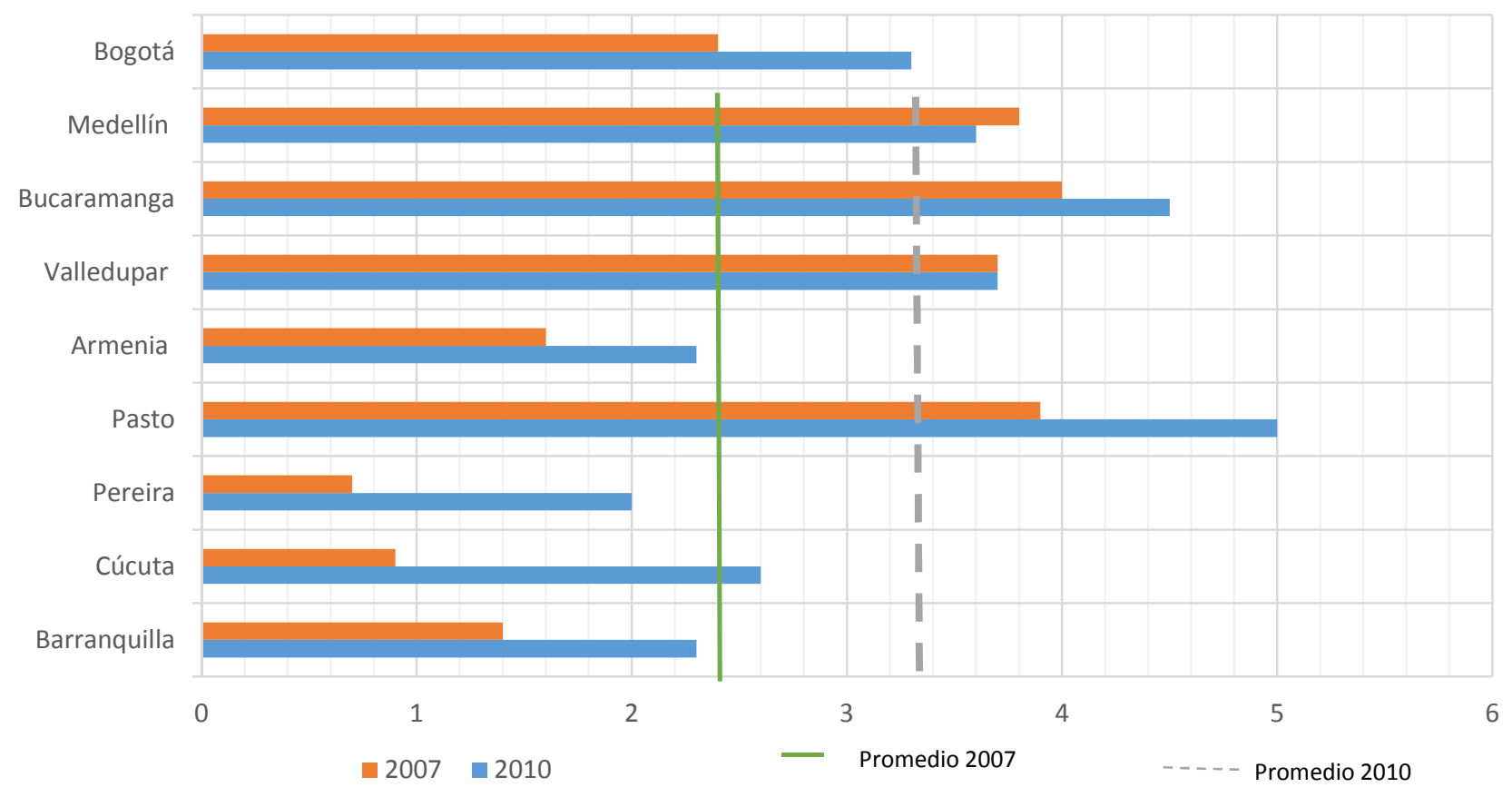

Figura 3. Índice de espacio público/ habitante en ciudades colombianas

Fuente: Municipios y Distritos. Elaboró: DNP-DDU (2010) 
puede lograr dando a conocer a los gobiernos de todo nivel del país su relación con el cumplimento de los $\mathrm{Ob}$ jetivos de Desarrollo Sostenible (ODS) y de los planes de desarrollo. Más de la mitad de los diecisiete ODS se hallan relacionados de alguna manera con las ciudades. Los espacios públicos verdes, a través del fortalecimiento de la cohesión y apropiación social, impactan directamente ocho de ellos (objetivos 1,2,3,10,12,13,15 y 16).

Se debería realizar una revisión detallada de los instrumentos de política pública a nivel nacional, con la intención de consolidar dichos instrumentos de carácter normativo, de obtener un impacto mayor y de facilitar la implementación de las estrategias. Una vez se realice esto, se pueden identificar las instituciones que se deben articular en los siguientes temas: reintegración de excombatientes, víctimas del conflicto armado, posconflicto, ordenamiento territorial, medio ambiente y salud.

Siendo un momento coyuntural, donde a nivel local se está dando una (re) programación de los planes de ordenamiento territorial, es el momento indicado para introducir la protección y la creación de nuevos espacios verdes urbanos, con un enfoque multidimensional y multifuncional, que permita garantizar los derechos humanos y generar equidad.

Se podrían desarrollar programas de pedagogía ciudadana que involucren el deporte, el arte y la cultura, así como la agricultura urbana, en los espacios verdes, con las diferentes comunidades que componen la ciudad (integrantes de la fuerza pública, desmovilizados, victimas, desplazados y el ciudadano que no ha tenido una relación directa con el conflicto), para generar en torno a estas actividades una sensibilización a las heridas del conflicto y empezar a reconstruir el tejido social.

Se pueden redefinir los espacios verdes como espacios de memoria y reconciliación para la no repetición de una historia de violencia. Por ejemplo, a través del arte en espacios verdes, se puede revindicar a las víctimas y fomentar la apropiación de estos espacios ciudadanos, lo que permitiría dar solución a la problemática identificada en el Conpes 3718 sobre la falta de apropiación colectiva de los espacios públicos.

Finalmente, se debería involucrar a la ciudadanía en los procesos de toma de decisiones, con la intención de fomentar la apropiación de los espacios verdes y su mantenimiento, pues es evidente que las estrategias desde las comunidades, apoyadas por las autoridades en una articulación coherente bottom-up -top-down, tienen una mayor perdurabilidad en el tiempo ya que generan identificación, satisfacción de las necesidades de la población y un reconocimiento con el territorio.

\section{Conclusión}

Al recoger las experiencias analizadas tanto de las sociedades en posconflicto, como las iniciativas ciudadanas y gubernamentales en Colombia, es preciso afirmar que los espacios verdes en las ciudades tienen un impacto significativo para el fortalecimiento de la cohesión social, además, permite entender los beneficios que las sociedades reciben de estas zonas en las ciudades, en donde no solo se fortalece el sentido de pertenencia a un lugar y se impacta positivamente la salud física y mental, sino que también tienen la capacidad de contar historias a través de los monumentos y crear nuevas experiencias y recuerdos de una sociedad en constante transformación. Con las iniciativas ciudadanas en torno a los espacios verdes, se evidenció que no solo prestan unos servicios a las sociedades de diversa índole, sino que se convierten en una oportunidad de transformación de las comunidades y especialmente de las vulnerables, pues ofrecen oportunidades de desarrollo integral y a largo plazo una evolución del estilo de vida de sus habitantes.

Para una sociedad como la colombiana, en donde el conflicto armado ha dejado huellas muy fuertes en sus habitantes, estos espacios en las ciudades dan la posibilidad de aliviar el estrés diario y brindan espacios de memoria y recordación tras 52 años de conflicto, como se evidenció en los espacios públicos del Salvador y Sudáfrica.

Una de las mayores problemáticas observadas es que la deficiencia del espacio público es una cuestión importante y no solo se da en Colombia, sino en los casos internacionales estudiados, sobre todo en las poblaciones y áreas de escasos recursos económicos, cuya densidad poblacional es exponencialmente más alta a las áreas con un ingreso mayor per cápita, lo que es una tendencia a nivel mundial. A pesar de las diferencias sustanciales entre los conflictos tomados como casos de estudio, cabe resaltar que se encontró como punto en común el pasado político oscuro de su historia y la capacidad de resiliencia de sus sociedades, en especial de la sociedad civil que se empoderó en el posconflicto y se apropió de los lugares de encuentro como las áreas verdes, para facilitar la transición y promover la dignidad humana, la diversidad y las libertades que brinda una nueva realidad.

Muchas de las iniciativas ciudadanas mostradas se tradujeron en políticas públicas que buscan articular el posconflicto con el desarrollo urbano a través de sus espacios públicos fortaleciendo los modelos bottom-up; sin embargo, estas medidas se quedan cortas en las áreas verdes, ya que no se aprovechan todos los servicios que pueden prestar y se limitan a la infraestructura construida en los espacios públicos.

Otra problemática observada relacionada con el desarrollo del artículo fue la falta de bibliografía e investigaciones que involucren las tres variables de análisis: espacios verdes urbanos, cohesión social y posconflicto. A pesar de que existen trabajos donde se ha contribuido a llenar este vacío de conocimiento y de práctica, las dimensiones ecológicas aún son desconocidas en las sociedades en posconflicto, debido a que el debate y las investigaciones en torno a la consolidación de la paz se han centrado 
en aspectos sociopolíticos. Y si bien se han desarrollado algunos trabajos relacionados con aspectos ambientales, estos se enfocan en el papel de las cuestiones ecológicas del medio ambiente y los recursos naturales, dejando de lado las preocupaciones por los problemas y procesos ambientales en el debate general de construcción de paz (Krampe, 2016). Por lo tanto, este artículo es una de las primeras aproximaciones al tema y deja abierto el debate para futuras discusiones académicas en torno a los diversos cuestionamientos que puedan surgir.

Por último, la naturaleza en las ciudades propicia espacios para la renovación personal y brinda herramientas de resiliencia social, lo cual se articula con las tendencias internacionales sobre sostenibilidad recogidas en los Objetivos de Desarrollo Sostenible y permite el cumplimiento de los planes de desarrollo a nivel nacional y local. Es por ello por lo que los espacios verdes son espacios públicos excepcionales y a su vez neutrales para la reconstrucción del tejido social, a través de políticas públicas enfocadas en los espacios verdes, que se construyan con la participación y el involucramiento de todos los ciudadanos, a fin de que realmente sean una herramienta efectiva de transformación, reconciliación y desarrollo integral de la sociedad colombiana.

\section{Referencias}

L'Escola de Cultura de Pau. (s.f). Construcción de paz: l'Escola de Cultura de Pau. Recuperado de http://escolapau.uab.cat/index. php?option=com_content $\&$ view $=$ article $\&$ id $=196 \% 3$ Aconstruccion-de-paz\&catid=70\&Itemid $=93 \&$ lang $=$ es

Amuprev. (S.f). Historia de éxito El Salvador: participación ciudadana en tardes de convivencia fortalecen prevención de la violencia en el municipio de Sonsonate; amuprev.org. Recuperado de http://www. amuprev.org/historias/historias/?id=64\#sthash.fFcoUgzp.dpuf

Aristóteles. (1932). Politica [Primera ed., N. Estévanez, Trad.] México: Casa Editorial Garnier Hermanos. Recuperado de http://biblio. juridicas.unam.mx/libros/2/766/pl766.htm

Ballesteros, J., Velásquez, C., Sierra, M., Vásquez, T. y Vélez, E. (2011). Santo Domingo Savio: un territorio reterritorializado. Territorios, 87-110.

Berroeta, H. y Moranta, T. (2012). La noción de espacio público y la configuración de la ciudad: fundamentos para los relatos de pérdida, civilidad y disputa. Polis, 57-80. Recuperado de http://polis. revues.org/3612

Borja, J., y Muxi, Z. (2003). El espacio público: ciudad y ciudadanía. Barcelona: Electa.

Buonomo Zabaleta, M. y Yanes, P. (2013). Crecimiento económico y cohesión social en América Latina y el Caribe. CEPAL.

Burbano, A. y Páramo, P. (2014). La ciudad habitable: espacio público y sociedad. Bogotá, D.C. : Universidad Piloto de Colombia.

Camelo, H. (2015). Aportes para la comprensión de la violencia en periodos de posconflicto. Ciudad paz-ando, 8-21.
Carmona, M. (2010). Contemporary public space, part two: CLassification . Journal of urban design, 175-173. https://doi. org/10.1080/13574801003638111

Chan, J., To, H. and Chan, E. (2006). Reconsidering social cohesion: Developing a definition and analytical framework for empirical research. Social indicators research, 75. https://doi.org/10.1007/ s11205-005-2118-1

Chetail, V. (2009). Post-Conflict Peacebuilding: A Lexicon. Oxford: Oxford University Press.

Código nacional de policía y convivencia. (2017). Colombia.

CONPES. (2012). CONPES 3718: Politica Nacional de Espacio Público. República de Colombia.

Corporación Alas de Mariposa. (S.f). Nosotros : Corporación Alas de Mariposa. Recuperado de http://www.alasdemariposa.com

Dawson, A. (2006). Geography of Fear: Crime an the transformation of public space in post-apartheid South Africa. En S. Low, \& N. Smith, The politics of Public Space (pp. 123-142). New York: Taylor $\&$ Fancis Group.

Di Pego, A. (2006). Pensando el espacio público desde Hannah Arendt. Un diálogo con las perspectivas feministas. Question. Recuperado de http://perio.unlp.edu.ar/ojs/index.php/question/article/view/222/161

Echandia, C. (2006). Dos décadas de escalamiento del conflicto armado en Colombia (1986-2006). Bogotá, D.C.: Universidad Externado de Colombia.

Ferry, J. M. (1995). Las transformaciones de la publicidad. En J. En Ferry, \& D. Wolton, El nuevo espacio público. Barcelona: Gedisa.

Forrest, R., \& Kearns, A. (2001). Social cohesion, social capital and the neighbourhood. . Urban studies, 38(12), 2125-2143. https://doi. org/10.1080/00420980120087081

González, M. y Carrizosa, C. (2011). Entre la planeación urbana, la apropiación del espacio y la participación ciudadana. Los pactos ciudadanos y el Parque Biblioteca España de Santo Domingo Savio. Estudios Politicos, 117-140.

Graham, L. F. (2012). Northern Ireland's approaches to Social Cohesion: A case study of social capital in victim support groups. Shared Space: A Research Journal for Peace, Conflict and Community Relations in Northern Ireland, 55-70.

Guerrero, E., y Culós, G. (2007). Indicadores ambientales en la gestión de espacios verdes. El parque Cerro La Movediza. Tandil, Argentina. Revista Espacios.

IDRD. (2015). Bogotá implementa estrategia de deporte para la paz: IDRD. Recuperado de http://www.idrd.gov.co/sitio/idrd/?q=node/1719

IGAC. (2014). Tan solo el 0,3 por ciento de todo el territorio: Comunidado de prensa. Recuperado de http://www.igac.gov.co/wps/

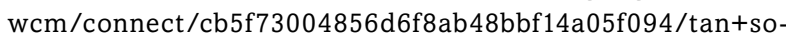
lo+el+0,3\%25+del+pais+es+urbano.pdf?MOD=AJPERES

Jiménez, A. (2010). La seguridad ciudadana en la transición gubernamental de El Salvador 2009. UFG Editores. 
Kazmierczak, A. (2010). Urban green spaces and social cohesion.

Kgalema, L. (1999). Symbols of hope: Monuments as symbols of remembrance and peace in the process of reconciliation. Centre for the Study of Violence and Reconciliation.

Krampe, F. (2016). Building Sustainable Peace: Understanding the Linkages between Social, Political, and Ecological Processes in Post-War Countries. Doctoral dissertation, Department of Peace and Conflict Research, Uppsala University. Report / Department of Peace and Conflict Research Uppsala University.

Lavaux, S. (2004). Degradacion ambienta y conflictos armados: Las conexiones. Bogotá, D.C.: Centro Editorial Universidad del Rosario.

Mantilla Valbuena, S. (2012). Economía y conflicto armado en Colombia: los efectos de la globalización en la transformación de la guerra. Latinoamérica. Revista de estudios Latinoamericanos, 35-73. Recuperado de http://www.scielo.org.mx/scielo.php?script=sci_arttext\&pid=S1665-85742012000200003\&lng=es\&tlng=es.

Masuku, T. (2015). A case study of the Irvory Park Community Work Programme. Centre for the Study of Violence and Reconciliation.

Millennium Ecosystem Assessment. (2003). Ecosystems and Human Well-Being. Recuperado de http://www.millenniumassessment. org/en/Framework.aspx

Montoya, J. (2017, 14 de marzo). Herramientas de biodiversidad en la planificación urbana en Colombia. Coloquio internacional interdisciplinario Colombia-Francia: La ciudad región sostenible como proyecto desafios actuales, visiones cruzadas y perspectivas. Bogotá, D.C.

Neira, H. (2007). La naturaleza del espacio público. Una visión desde la filosofía. En O. Segovia, espacios públicos y construcción social, hacia un ejercicio de ciudadanía (pp. 29-40). Santiago de Chile : Ediciones SUR.

ONU. (1992). UN documents. Recuperado de Preventive diplomacy, peacemaking and peace-keeping. : http://www.un-documents. net/a47-277.htm

OPAMSS. (S.f). Políticias públicas metropolitanas: Oficina de Planeación del Área Metropolitana de San Salvador. Recuperado de http:// www.opamss.org.sv/index.php?option=com_content\&view $=$ article\&id=15:gestion-institucional\&catid=10:que-hacemos\&Ite$\operatorname{mid}=140$

Ordóñez, C. and Duinker, P. (2014). Urban Forest Values of the Citizenry in Three Colombian Cities. Society \& Natural Resources: An International Journal, 834-849. https://doi.org/10.1080/089419 20.2014 .905891
Orsini, F., y Montoya, J. (2016). Infraestructura urbana ecológica: planes municipales integrales en el Golfo de Urabá. En M. A. Mejía, Naturaleza Urbana. Plataforma de Experiencias. Bogotá, D.C.: Instituto Humboldt.

Osorio, F. (2007). Allá se sufre mucho... pero se vive mejor, identidades campesinas desde lo perdido: los desplazados y sus percepciones. En N. R. Rosario Documento presentado en el XI Congreso de Antropología en Colombia. Bogotá, D.C.: (ICANH).

Palmary, I. (2015). Reflections on social cohesion in contemporary South Africa. Psychology in Society, 62-69. Recuperado de http:// www.scielo.org.za/pdf/pins/n49/05.pdf

Peters, K., Elands, B. and Buijs, A. (2010). Social interactions in urban parks:Stimulating social cohesion? Urban Forestry \& Urban Greening, 93-100. https://doi.org/10.1016/j.ufug.2009.11.003

Rettberg. (2013). A la construcción de paz bajo la lupa: una revisión de la actividad y de la literatura académica internacional. Estudios Políticos (42), 13-36.

Rettberg, A., Camacho, A., Chaux, E., García, A., Iturralde, M., Sánchez, F. (2002). Preparar el futuro: conflicto y post-conflicto en Colombia (Vol. 20). Alfaomega.

Reyes, V. (2016). La anomia. Espacios, tiempos, y conflictos anómicos. Análisis de caso. Bogotá, D.C.: Ediciones Aurora.

Ruiz, N. Y. (2009). Colombia se consolida como una sociedad urbana. En En M. C. Herrera, Procesos Urbanos Informales y Territorio: Ensayos en torno a la construcción de la Sociedad, Territorio y Ciudad (pp. 113-122). Bogotá, D.C.: Universidad Nacional de Colombia.

Segovia, O. (2007). Espacios públicos y construcción social. Hacia un ejercicio de ciudadanía. Ediciones SUR.

Tepper, J. (S.f). Reconciling Urban Spaces in post-Apartheid South Africa Glimpses of Cape Town's 'liberated' landscape. Durkheimian Explanation for Suicide Terrorism. Recuperado de https:// www.researchgate.net/publication/237481885_Reconciling_ Urban_Spaces_in_post-Apartheid_South_Africa_Glimpses_of_ Cape_Town's_'liberated'_landscape

United Nations. (Octubre de 2016). United Nations Conference on housing and sustainable Urban Development. Habitat III. Quito.

Veit, A., Barolsky, V. and Pillay, S. (2011). Violence and violence research in Africa South of the Sahara. International Journal of Conflict and Violence.

Williams, J. (2000). South Africa: urban transformation. Cities, 167183. https://doi.org/10.1016/S0264-2751(00)00012-3 